\title{
Andrographolide nanoparticles in leishmaniasis: characterization and in vitro evaluations
}

This article was published in the following Dove Press journal:

International Journal of Nanomedicine

9 December 2010

Number of times this article has been viewed

\section{Partha Roy' \\ Suvadra Das' \\ Tanmoy Bera ${ }^{2}$ \\ Subhasis Mondol ${ }^{2}$ \\ Arup Mukherjee'}

'Department of Chemical Technology, University of Calcutta, Kolkata, West Bengal, India; ${ }^{2}$ Department of Pharmaceutical Technology, Ja davpur University, Kolkata, West Bengal, India
Correspondence: Arup Mukherjee Department of Chemical Technology, University of Calcutta, 92, A.P.C. Road, Kolkata-700009, West Bengal, India

Tel +9l-33-23508386;

$+91-33-23508387$

Fax +9I-33-235I9755

Email arupm1234@gmail.com

\begin{abstract}
Andrographolide (AG) is a diterpenoid lactone isolated from the leaves of Andrographis paniculata. AG is a potent and low-toxicity antileishmanial agent. Chemotherapy applications of AG are, however, seriously constrained because of poor bioavailability, short plasma half-life, and inappropriate tissue localization. Nanoparticulation of AG was therefore envisaged as a possible solution. AG nanoparticles (AGnp) loaded in 50:50 poly(DL-lactide-co-glycolic acid) were prepared for delivery into the monocyte-macrophage cells infested with the amastigote form of leishmanial parasite for evaluation in the chemotherapy of leishmaniasis. Particle characteristics of AGnp were optimized by proportionate application of a stabilizer, polyvinyl alcohol (PVA). Physicochemical characterization of AGnp by photon correlation spectroscopy exhibited an average particle size of $173 \mathrm{~nm}$ and zeta potential of $-34.8 \mathrm{mV}$. Atomic force microscopy visualization revealed spherical nanoparticles with a smooth surface. Antileishmanial activity was found to be significant for the nanoparticle preparation with $4 \%$ PVA $\left(\mathrm{IC}_{50} 34 \mu \mathrm{M}\right)$ in about one-fourth of the dosage of the pure compound $\mathrm{AG}\left(\mathrm{IC}_{50} 160 \mu \mathrm{M}\right)$. AGnp therefore have significant potential to target the infested macrophage cells and prove valuable in chemotherapy of neglected tropical diseases such as leishmaniasis.
\end{abstract}

Keywords: andrographolide, nanoparticles, antileishmanial chemotherapy

\section{Introduction}

Leishmaniasis is a neglected tropical disease (NTD) caused by protozoan parasites belonging to the genus Leishmania. The disease is currently endemic in 88 countries of the developing world and showing increasing prevalence in immunosuppressive conditions such as HIV/AIDS. ${ }^{1}$ The World Health Organization has put out an ardent appeal for the development of drugs and delivery devices against leishmaniasis. ${ }^{2}$ Therapeutic strategy today is limited to pentavalent antimony compounds such as meglumine antimoniate and sodium stibogluconate $(\mathrm{SbV})$ with later stage application of amphotericin B (AmB) and pentamidine. The efficacy of organic antimonials is rapidly declining because drug resistance is increasing, while both $\mathrm{AmB}$ and pentamidine are associated with serious toxic side effects. ${ }^{3,4}$ Although a liposomal delivery device for $\mathrm{AmB}$ addresses some of its toxic side effects, the formulation is prohibitively expensive and has liposomal instability problems. Increasing lipid load in the treatment of systemic leishmaniasis is an added concern. Paromomycin and miltefosine are two very recent developments that have been intensely evaluated as alternative compounds in leishmaniasis chemotherapy. ${ }^{4,5}$ Development in leishmaniasis chemotherapy is, however, far too little, mostly due to very low specificity, high cost, and high drug toxicity. One approach to antileishmanial chemotherapy could be 
a systematic application of functional drug delivery devices carrying locally available low-cost chemotherapeutics which have alternative modes of action.

Andrographolide (AG), a labdane diterpenoid extracted from the leaves of the Indian medicinal plant Andrographis paniculata, is a strong antiparasitic and antileishmanial compound. ${ }^{6}$ Like most diterpenes, AG is sparingly soluble in water, ${ }^{7}$ limiting its biodistribution and localization. Additionally, AG is unstable in extremes of gastrointestinal alkaline and acidic conditions and has a very short biological half-life ( $t_{1 / 2}=2$ hours). ${ }^{8}$ Development of a suitable delivery device for the diterpines such as AG can therefore provide a relatively low cost, indigenous therapeutic lead in NTDs such as leishmaniasis.

This work focuses on the preparation of AG nanoparticles (AGnp) primarily to improve uptake in macrophage cells infected with the leishmanial pathogenic amastigote form. The Food and Drug Administration (FDA)-approved biopolymer for nanoparticulation, poly(DL-lactide-coglycolic acid) (PLGA), has an edge for its long stabilization in circulation. ${ }^{9}$ Prolonged-release AG nanoparticles have been successfully prepared with the proportionate use of polyvinyl alcohol (PVA) as stabilizer and using different formulation variables. AGnp have macrophage-specified localization and have proved encouragingly efficacious in infective forms of leishmaniasis.

\section{Experiment}

\section{Materials and facilities}

Standard Borosil ${ }^{\circledR}$ glassware (Mumbai, India) was used for preparation and analysis experiments. A Vibra cell VCX 750 $700 \mathrm{MW}$ sonicator (Sonics, Newtown, CT), a homozinizer model TH 02 (Omni International, Kennesaw, GA), a Mettler-Toledo AL54 0.00001-g precision balance (Mettler, Columbus, $\mathrm{OH}$ ), a Tarsons Accupipet (Tarsons, Calcutta, India), and a Himac CS120GHXL ultracentrifuge (Hitachi Koki, Tokyo, Japan) were used in preparative processes. Chemical analyses were carried out in a Waters dual pump HPLC model 515 (Waters, New Castle, DE). A zetasizer Nano ZS (Malvern Instruments, Malvern, UK), a Nanoscope $3 \mathrm{~A}$ atomic force microscope (Veeco, Plainview, NY), a FT-IR 670 Plus (Jasco, Tokyo, Japan), and a fluorescence microscope (BX51, Olympus, Center Valley, PA) were used for most particle characterization and imaging experiments. All solvents used were of HPLC grade and of E. Merck or Spectrochem brand. Water used was of millipore grade, Elix 3 Century ZLXS5003Y (Millipore, Billerica, MA). PLGA (50:50, MW 40,000-75,000), dialysis tubing D9652
(MW cut off 12,400), FITC (fluorescein 5(6)-isothiocyanate), PVA (89,000-98,000), and RPMI-1640 cell culture medium were purchased from Sigma-Aldrich (St. Louis, MO). Andrographolide was purchased from Natural Remedies (Bangalore, India) while $\mathrm{SbV}$ was a generous gift from Albert David Ltd. (Kolkata, India) and AmB was purchased from Sigma-Aldrich. Windows Excel 2003 version and Sigmaplot 6.0 (Zendal Scientific, USA) were used for the data analysis.

\section{Methods}

\section{Preparation of AGnp}

AG nanoparticles were prepared following an emulsion solvent evaporation technique. A combination of both sonication and homogenization was used to increase shear stress during emulsification to produce small size particles with higher drug loading. Briefly, $50 \mathrm{mg}$ PLGA and $2.5 \mathrm{mg}$ of AG were dissolved together in $3 \mathrm{~mL}$ of chloroform. This phase was emulsified by sonication for 1 minute in $12 \mathrm{~mL}$ of different concentrations $\mathrm{w} / \mathrm{v}$ ( $2 \%$ Formulation A, $4 \%$ Formulation B, and $6 \%$ Formulation C) of aqueous PVA at $20 \mathrm{kHZ}$. The resultant oil/water emulsion was homogenized for 20 minutes at 20,000 rpm under external cooling in ice water. The solvent evaporation was continued for 12 hours over a magnetic stirrer at room temperature. The nanoparticles (AGnp) formed were harvested by ultracentrifugation at $30,000 \mathrm{rpm}$ for 25 minutes at $4^{\circ} \mathrm{C}$. AGnp were washed with water, recentrifuged, and preserved in vacuum desiccators at $4^{\circ} \mathrm{C}$ until further evaluation. Each formulation was prepared in quadruplicate for analysis in later stages (Table 1).

Fluorescent PLGA nanoparticles (FAGnp) were prepared in a similar fashion where FITC was used in place of AG.

\section{Particle size and polydispersity}

The particle size of AGnp preparations was measured in a zetasizer Nano ZS against a 4 mw He-Ne laser beam, $633 \mathrm{~nm}$, and back scattering angle of $173^{\circ}$. Aliquots from each preparation batch were sampled in cuvettes and particle size along with polydispersity index (PDI) were determined.

\section{Zeta potential}

Zeta potential of the AGnp preparations was measured in a zetasizer set up. Aliquots from preparation batches were sampled in disposable zeta cells and zeta potentials were determined on the basis of electrophoretic mobility under an applied electrical field. 
Table I Characterization of AGnp preparations

\begin{tabular}{lllll}
\hline $\begin{array}{l}\text { AGnp } \\
\text { preparations }\end{array}$ & $\begin{array}{l}\text { Particle } \\
\text { size }(\mathbf{n m})\end{array}$ & $\begin{array}{l}\text { Zeta potential } \\
(\mathbf{m V})\end{array}$ & PDI & $\begin{array}{l}\text { Entrapment } \\
\text { efficiency \% }\end{array}$ \\
\hline Formulation A & $426 \pm 21.2^{*}$ & $-22.2 \pm 2.1$ & $0.155 \pm 0.03$ & $59 \pm 4.4^{* *}$ \\
Formulation B & $173 \pm 12.6^{*}$ & $-34.8 \pm 1.4$ & $0.229 \pm 0.01$ & $80 \pm 3.5^{*}$ \\
Formulation C & $225 \pm 18.4$ & $-34.2 \pm 1.8$ & $0.468 \pm 0.02$ & $56 \pm 5.2$ \\
\hline
\end{tabular}

Notes: Results expressed as mean $\pm S D(n=4)$; $P<0.0$ I significant difference compared with Formulation $C$; ${ }^{*} P<0.5$ no significant difference compared with Formulation $C$. Abbreviations: AGnp, andrographolide nanoparticles; PDI, polydispersity index.

\section{Atomic force microscopy}

AGnp suspensions $(100 \mu \mathrm{L})$ in water were deposited onto fused mica substrates. The particles were visualized in atomic force microscopy (AFM) in tapping mode using an RTESP tip with 267 to $328 \mathrm{kHz}$ resonance frequency at a scan speed of $1.2 \mathrm{~Hz}$.

\section{Fourier transform infrared (FTIR) studies}

FTIR studies were carried out to observe any AG and biopolymer interactions. AG, a blend of AG with PLGA, and PVA and a blend of PLGA and PVA were diluted separately with IR grade $\mathrm{KBr}$ in the ratio 1:100 and were pelletized (1000 psi). The pellets were scanned over a wave number range of 4000 to $400 \mathrm{~cm}^{-1}$ and the data stacked in Bio-Rad KnowItAll (Bio-Rad, Hercules, CA) software to compare and search for any chemical interactions.

\section{AG entrapment efficiency (\%)}

All AG estimations were carried out in a reverse phase HPLC system. The mobile phase was acetonitrile: $0.1 \%$ $(\mathrm{v} / \mathrm{v})$ phosphoric acid in water $(40: 60, \mathrm{v} / \mathrm{v})$ at a flow rate of $1 \mathrm{~mL} / \mathrm{min}$. The analysis was carried out using a $\mathrm{C}_{18}$ column (Supelco, Bellefonte, PA), $25 \times 4.6 \mathrm{~mm}$, and a PDA detector (2996, Waters). A peak area (y) vs concentration (x) graph for AG was first prepared, $\mathrm{y}=28051 \mathrm{x}+51747$, $R^{2}=0.9979$; retention time was 4.5 minutes. This was used to detect $A G$ concentrations throughout. Mass of $A G$ in solution before and after nanoparticulation in supernatant was determined in HPLC experiments for calculation of entrapment efficiencies. ${ }^{10}$

AG entrapment $(\%)$

$$
=\left(\begin{array}{l}
\text { Mass of AG originally taken } \\
\text { - Mass of AG in supernatant } \\
\text { Mass of AG originally taken }
\end{array}\right) \times 100
$$

\section{In vitro release studies}

AGnp equivalent to about $2 \mathrm{mg}$ of $\mathrm{AG}$ load were dispersed in $1 \mathrm{~mL}$ of phosphate buffer (100 mM, pH 7.4) and the dispersion was carefully washed into a dialysis bag. The dialysis bags were placed in glass vials containing $10 \mathrm{~mL}$ of phosphate buffer and maintained at $37^{\circ} \mathrm{C}$ in a shaker bath. At predetermined time intervals, the release medium in vials was taken out and replaced with fresh buffer medium. The amount of AG released per timed sample was estimated in HPLC. A standard curve plot was used for analysis with necessary corrections for the dilution factors. The in vitro release and kinetics for all three types of preparations were similarly studied. In order to understand the molecular release mechanism, the Korsemeyer-Peppas model was applied and $\mathrm{n}$ and $\mathrm{K}$ values were calculated using Sigmaplot 6.0 software.

\section{Nanoparticle uptake by fluorescence microscopy}

In order to study nanoparticle uptake, macrophages $\left(4 \times 10^{5}\right.$ cells/well) were seeded with FAGnp $(0.5 \mathrm{mg} / \mathrm{mL})$, incubated for 2 to 4 hours, and observed in FITC channel under a fluorescence microscope.

\section{Assessment of antileishmanial activity} Parasites and macrophages

Leishmania donovani AG 83(MHOM/IN/1983/AG83) ${ }^{11,12}$ was isolated from an Indian patient with kala-azar. Macrophages were collected by peritoneal lavage from mice (Swiss albino, female $22 \pm 2 \mathrm{~g}$ ) given with intraperitoneal injection of $0.5 \mathrm{~mL}$ (4\%) of thioglycolate broth 5 days before harvest. Cells were grown on RPMI-1640 medium supplemented with $2 \mathrm{~g} / \mathrm{L} \mathrm{NaHCO}_{3}, 10 \%$ fetal calf serum, $100 \mathrm{U}$ penicillin, and $50 \mu \mathrm{g}$ streptomycin $/ \mathrm{mL}$. Exudate cells were harvested from the peritoneal cavity in RPMI-1640 medium and plated onto tissue-culture dishes with $35 \mathrm{~mm}$ diameter wells. After incubation for 2 hours at $37^{\circ} \mathrm{C}$, nonadherent cells were removed by thorough washing. The remaining adherent macrophages were cultured for 24 hours at $37^{\circ} \mathrm{C}$ in an incubator $\left(5 \% \mathrm{CO}_{2} /\right.$ air) for further studies. Cultures prepared in this manner contained $\geq 95 \%$ macrophages, as evaluated by their ability to take up latex beads and by morphology. 
L. donovani amastigotes were grown and maintained as described by Debrabant et al..$^{13}$ Axenically grown amastigotes were maintained at $37^{\circ} \mathrm{C}$ with $5 \% \mathrm{CO}_{2} /$ air by weekly subpassages in MAA/20 (medium for axenically grown amastigotes) at $\mathrm{pH} 5.5$ in $177 \mathrm{~cm}^{2}$ petri dishes. ${ }^{14}$ Under these conditions, promastigotes differentiated to amastigotes within 120 hours. Cultures were maintained by 1:3 dilutions once a week.

\section{Axenic amastigote AG susceptibility assay}

Susceptibility determinations of $L$. donovani axenic amastigotes were carried out using a modification of the promastigote direct counting growth inhibition assay. ${ }^{15}$ Amastigotes were seeded at an initial concentration equivalent to early log phage $\left(3 \times 10^{5}\right.$ amastigotes $\left./ \mathrm{mL}\right)$ and allowed to multiply for 72 hours either in medium alone or in presence of serial dilutions of drug or inhibitor until late log phage $\left(1 \times 10^{6}\right.$ cells $\left./ \mathrm{mL}\right)$. Axenic amastigote numbers doubled 3 to 4 times during the assay. AG susceptibility experiments were performed in the maintenance media. The $50 \%$ inhibitory concentration $\left(\mathrm{IC}_{50}\right)$ for the test drug was determined. Amastigotes were counted microscopically using a hemocytometer after being passed 3 times through a 27 -gauge needle in order to separate any clumps, as part of a standard procedure and cell determination accuracy. All experiments were done in triplicate.

\section{Amastigote in macrophage AG susceptibility assay}

AG susceptibility of $L$. donovani was determined by following a modification of the method of Chang. ${ }^{16}$ Briefly, macrophages were seeded at $4 \times 10^{4}$ cells/well in $\alpha-10$ medium with chamber slides. Following a day treatment at $37^{\circ} \mathrm{C}$ to allow attachment, macrophages were infected with axenic amastigotes $\left(4 \times 10^{5}\right.$ amastigotes/well) in $\alpha-10$ medium and then incubated for 4 hours at $37^{\circ} \mathrm{C}$ to allow infection. The chamber slides were washed once with Dulbecco's phosphate-buffered saline. AG solutions diluted in $\alpha-10$ medium were added to the appropriate wells and the slides were incubated at $37^{\circ} \mathrm{C}$ for a further 72 hours before staining with Giemsa. Medium and AG solutions were replaced with fresh ones every 24 hours. The initial infection was determined by fixing 2 wells with Giemsa immediately following a 4-hour incubation. Control wells of infected macrophages were incubated in medium alone to determine the doubling times of amastigotes in macrophages over the experimental duration ( 72 hours). The viabilities of AG treated or untreated macrophages were also assessed at 72 hours. Experiments were done in triplicate. The initial percentage of macrophages infected with amastigotes was determined by microscopic inspection (100x) of the cells. $\mathrm{IC}_{50}$ of the test solutions was determined based on the value of number of amastigote per macrophage.

\section{Cytotoxicity against mammalian macrophages}

Macrophages cells were cultured in minimum essential medium (MEM, Gibco), supplemented with $20 \mathrm{mM}$ L-glutamate, $16 \mathrm{mM} \mathrm{NaHCO}_{3}, 5 \%$ fecal calf serum and penicillin-streptomycin. The assay was performed in 96-well tissue culture plates in the presence of standard counts of macrophages. The wells were seeded with test solutions and the viable macrophages were counted microscopically.

\section{Statistical analysis}

Experimental results were expressed as mean \pm standard deviation. Student's $t$-test was used to calculate the statistical difference of mean values. Differences were considered significant at $P<0.01$.

\section{Results}

Limited solubility of AG was a major constraint in the preformultion stages. Ethanolic solution of AG caused precipitation of the biopolymer in the emulsification stage. AG dissolved in DMSO and ethyl acetate failed to load, because of higher loss of diffusive mass. Chloroform was used as a solvent as it can keep both AG and PLGA together in solution and enable optimization experiments in particle size and loading. ${ }^{17}$ Three categories of AGnp were prepared varying in stabilizer PVA concentration (Table 1). Nanoparticles prepared in the sonication and homogenization procedure were evenly distributed and relatively small. In other experiments, initially when only a homogenization step was used for dispersion, larger particle size, irregular drug load, and skewed particle distribution were observed. ${ }^{18}$ PLGA nanoparticle preparations in a sequential application of sonication and homogenization provided better control of particle sizing and drug loading. A clear stable dispersion of AGnp in water with a color tint due to AG loading was obtained (Figure 1).

\section{Nanoparticle size and zeta potential}

The particle size, PDI, and zeta potential for all AGnp preparations were studied in PCS and the results are presented in Table 1. PVA stabilizer appeared to have a distinct role both in nanoparticle size and PDI. Formulation A, having low PVA concentration of $2 \%$, produced larger size particles of $426 \mathrm{~nm}$ and a much reduced PDI of 0.155. In Formulation B, particle size was around $173 \mathrm{~nm}$, when $4 \% \mathrm{w} / \mathrm{v}$ of PVA was used. 

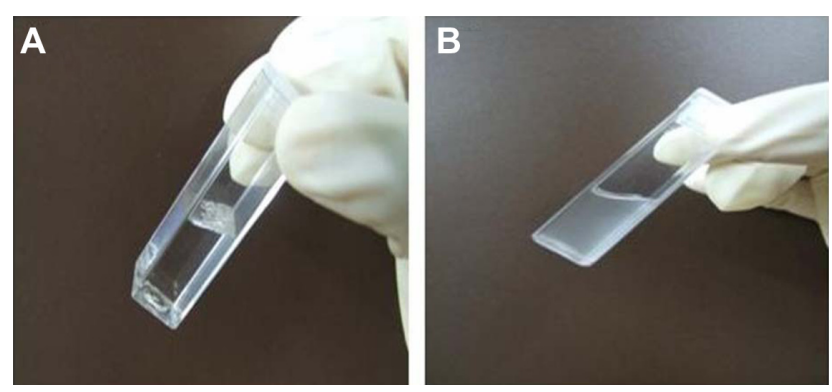

Figure I Andrographolide (AG) and andrographolide nanoparticles (AGnp) in water medium. A) AG flakes insoluble in water. B) AGnp (Formulation B) colloid in water.

Increasing the concentration of PVA had an oscillating effect, and in Formulation C where 6\% w/v PVA was used, particles were $225.3 \mathrm{~nm}$ and PDI was 0.468 with little skewness in particle distribution. Formulation B had a zeta potential of $-34.8 \mathrm{mV}$ with a near perfect Gaussian particle distribution. The observations were consistent with earlier findings. ${ }^{18}$

\section{AFM surface topography}

AFM was extensively used to understand the particle surface topography for AGnp. The 3D amplitude data of every AGnp preparation exhibited a spherical shape with a smooth solid surface and minimal coalescence. Figure 2 shows an AFM study of an observation zone and 3D surface characteristics of individual particles from Formulation B.

\section{FTIR studies for exipient interactions}

Because AG is a delicate phytoconstituent, prior study was important to determine if there was any type of chemical interaction with the biopolymer materials used in nanoparticulation.

FTIR scan for AG was typical and the AG O-H stretching vibrations appeared at $3402 \mathrm{~cm}^{-1}$, indicative of an intermolecularly hydrogen bonded structure. $\mathrm{C}-\mathrm{O}$ stretching appeared at $1030 \mathrm{~cm}^{-1}$ and at $1078 \mathrm{~cm}^{-1}$ for the primary and
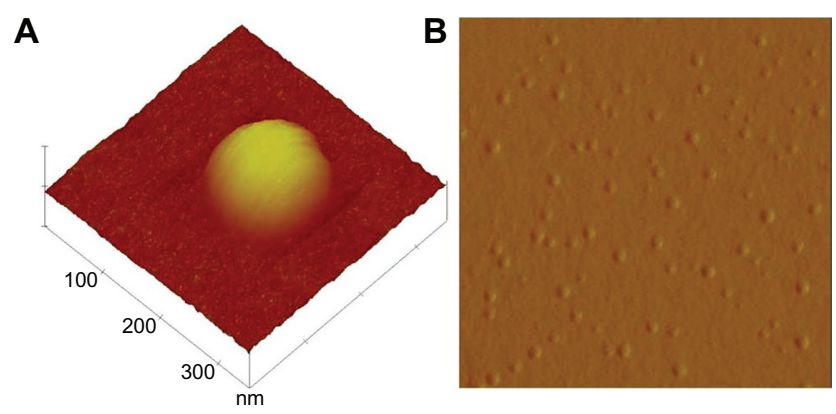

Figure 2 Atomic force microscopy study of andrographolide nanoparticles. A) Single particle 3D surface topography. B) 2D study of particle distribution (Formulation B). the secondary alcoholic functions. Corresponding in plane $\mathrm{O}-\mathrm{H}$ bending responded at $1420 \mathrm{~cm}^{-1}$ and the characteristic AG lactone responded at $1727 \mathrm{~cm}^{-1}$ (Figure 3). ${ }^{19}$ PLGA and PVA biopolymers individually and when blended together responded at $2935 \mathrm{~cm}^{-1}$ due to linear $\mathrm{CH}_{2}$ stretching and at $1756 \mathrm{~cm}^{-1}$ due to the ester bond, respectively. The biopolymer blend did not show any absorbance in specific regions of AG reactive functional groups. ${ }^{20}$ When $\mathrm{AG}$ was blended with PLGA and PVA and scanned in FTIR, all AG functional groups remained intact in position, showing that there was no appreciable chemical interaction between $A G$ and the biopolymer materials used.

\section{Nanoparticle drug entrapment}

Effectiveness of a drug delivery device is directly dependent on its drug payload. Payload efficiency, often termed entrapment efficiency, is an important determinant for preparation process efficiencies. AG entrapment in PLGA was observed in quadruplicate for all formulations (Table 1). Formulation B showed the highest entrapment efficiency of $80 \pm 3.5 \%$.

\section{In vitro drug release and release kinetics}

The time-dependent in vitro release of AG was studied, and the average percentage cumulative release over time is plotted in Figure 4. The release responses for all formulations were biphasic in nature. The initial release (burst effect) was fast, indicative of some nanoparticle surface-adsorbed AG molecules. The release, however, remained fast for a prolonged period of 720 hours.

The faster release rate was recorded in the initial 48 hours followed by a sustained release period for the remaining $A G$ payload. In Formulation A, however, almost $65 \%$ of payload was released in media in 48 hours, indicating that the concentration of PVA used is important not only for PLGA particle sizing but also to enable drug entrapment. ${ }^{18}$ Formulation B therefore appeared most promising because of its superior drug load, appropriate Gaussian particle distribution, and sustained release over a sufficiently prolonged period.

The order kinetics for all preparations was statistically evaluated. AG in vitro release for Formulation B was observed following zero order kinetics, consistent with prolonged-release particulate drug delivery devices. Understanding the payload release mechanism for particulate delivery systems is an important criterion for further correlations. The Korsemeyer-Peppas model (equation 1) describes the release mechanism of particulate delivery devices over a time range. ${ }^{21}$

$$
\mathrm{Mt} / \mathrm{M} \alpha=\mathrm{Kt}^{\mathrm{n}}
$$



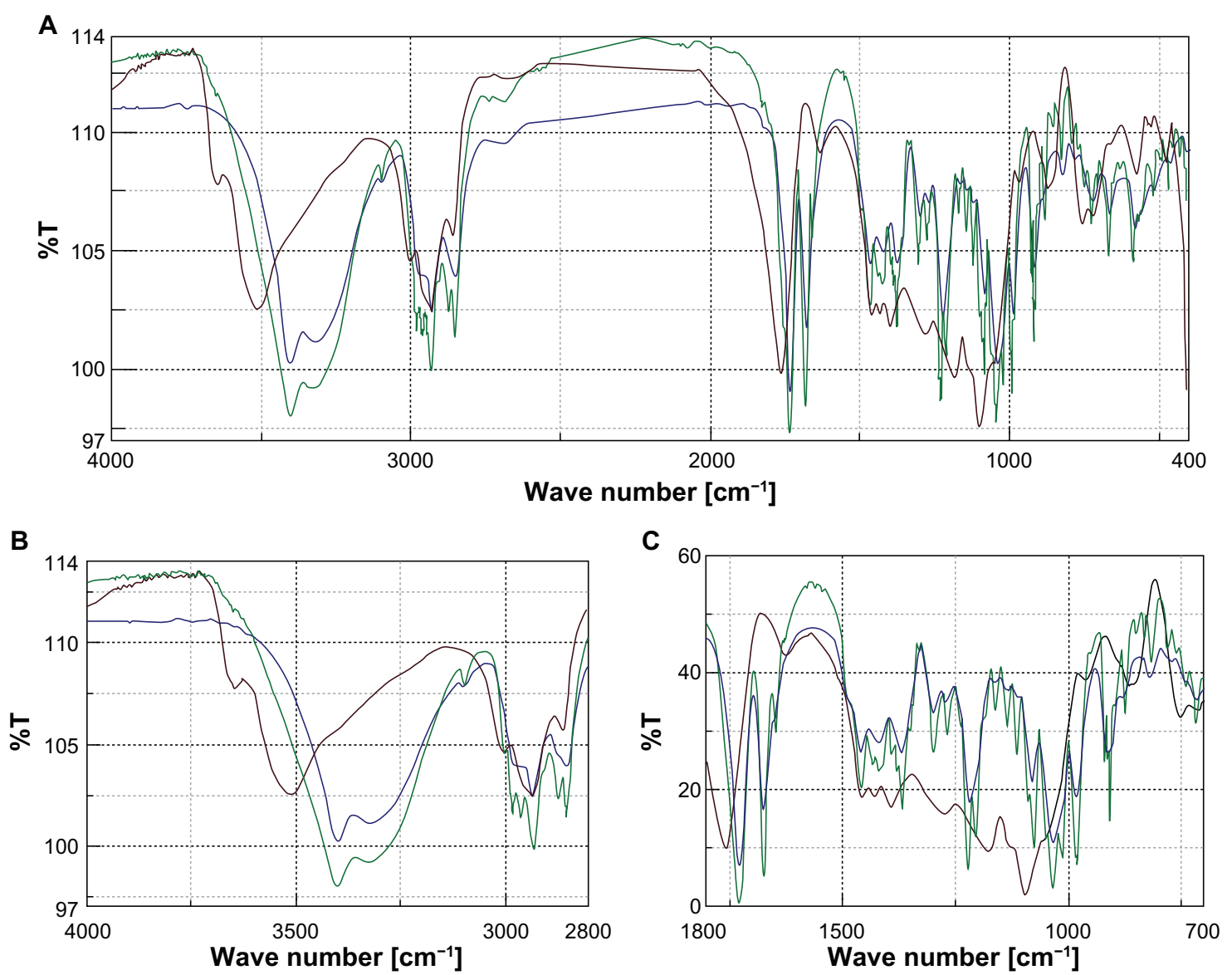

Figure 3 A) Fourier transform infrared overlay of entire region. B) Overlay at $\mathrm{C}-\mathrm{OH}$ region. C) Overlay at $\mathrm{C}-\mathrm{OH}$ region and $\mathrm{CH} 2$ region. Color codes: andrographolide (AG), blue; polyvinyl alcohol/poly(DL-lactide-co-glycolic acid) (PVA/PLGA), brown; AG/PVA/PLGA, green.

Where, Mt, mass released at time point ' $\mathrm{t}$ ', M $\alpha$, total mass load, $\mathrm{n}$ is the release exponent, and $\mathrm{K}$ is the constant accommodating the structural and geometric features.

AG mass release for Formulation B nanoparticles provided a good fit (Table 2). The $\mathrm{n}$ value recorded was 0.33 , indicating the release mechanism is not a simple Fickian diffusion ${ }^{22}$ for AGnp. This could appear when mass release is associated with slow polymer surface erosion or a bulk mass penetration. AFM studies were carried out separately on particles sampled from after 360 hours of exposure in release media. Nanoparticles sampled after 600 hours of release evidenced distinct erosion on PLGA polymer surfaces (Figure 5). This clearly explained surface erosion associated with in vitro release mechanism in later hours of AG release.

\section{In vitro antileishmanial activity and cytotoxicity}

The susceptibilities of both the axenic and intracellular amastigotes to AG and 3 AGnp preparations were evaluated following standard protocols. $\mathrm{The} \mathrm{IC}_{50}$ values in each case were compared against a known antileishmanial compound, $\mathrm{SbV}$. AG itself was a superior antileishmanial to $\mathrm{SbV}$ both in axenic amastigote and in vitro macrophage infective forms. Additionally, cytotoxicity due to AG was at least half that of $\mathrm{SbV}$ (Table 3). AGnp in Formulation B was, however, almost 5 times as efficacious as $\mathrm{AG}$ and about 8 times that of $\mathrm{SbV}$ in macrophage infective forms of amastigotes. Superior efficacies of AGnp were primarily attributed due to their improved localization in macrophage cells. Additionally, the small negative surface charge on nanoparticles prepared provided a colloidal dispersion that was conducive to entrapment onto nonflagellated amastigotes. A clear order in potency can therefore be shown as Formulation B $>$ Formulation $\mathrm{A}>$ Formulation $\mathrm{C}>\mathrm{AG}>\mathrm{SbV}$. AmB, a strong antileishmanial antibiotic, was also evaluated. Leishmanicidal activity of AmB was profound and was similar in axenic amastigote and microphage infested amastigotes, $\left(\mathrm{IC}_{50}\right.$ $0.2 \pm 0.1 \mu \mathrm{M}$ and $0.15 \pm 0.1 \mu \mathrm{M}$, respectively). AmB was, however, exceedingly cytotoxic, $50 \%$ cytotoxicity concentration $\left(\mathrm{CC}_{50}\right)>13 \mu$ mole, while $\mathrm{CC}_{50}$ due to $\mathrm{AG}$ was $>10^{3}$ $\mu$ mole and that of Formulation B was $>2 \times 10^{3} \mu$ mole. 


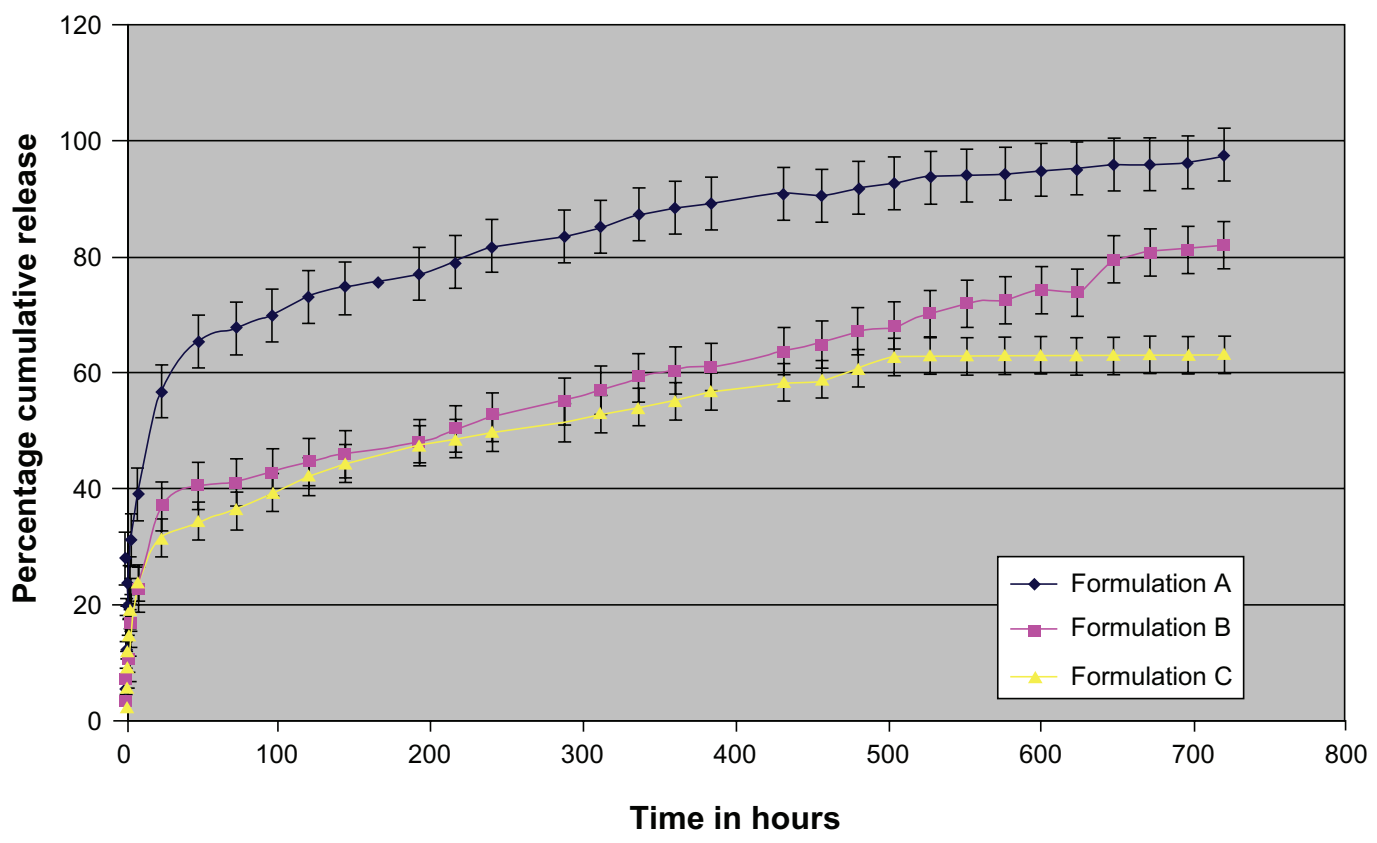

Figure 4 In vitro release profile of andrographolide nanoparticle preparations. Results expressed as mean $\pm S D(n=3)$.

A separate set of experiments with FITC-loaded nanoparticles prepared similarly showed a considerable macrophage uptake, peaking within 2 hours of incubation as observed under fluorescence microscopy (Figure 6). Empty nanoparticles without any AG load, when tested both for cytotoxicity and antileishmanial activity, have proved sufficiently innocuous even up to the tested dosage of $110 \mu \mathrm{g} / \mu \mathrm{L}$. AGnp antileishmanial activity was attributed to the $A G$ payload and to macrophage localization.

\section{Discussion}

Chemotherapy is the principal option in treatment of leishmaniasis. ${ }^{23}$ The parasitic disease is presently showing increasing prevalence due to multiple factors, including resistance to traditional chemotherapeutic agents, difficulties in drug localization, worsening immune environment in developing countries, and population migration and prevalence of other infective immune diseases such as HIV/AIDS. Prohibitive cost and the time lag in development of new chemotherapeutics are other hurdles.

Table 2 Korsemeyer-Peppas release kinetics modeling for AGnp

\begin{tabular}{lll}
\hline $\begin{array}{l}\text { AGnp } \\
\text { preparation }\end{array}$ & $\begin{array}{l}\text { Korsemeyer-Peppas } \\
\text { model parameters }\end{array}$ \\
\hline Formulation A & $\mathrm{n}=0.26$ & $\mathrm{~K}=0.19$ \\
Formulation B & $\mathrm{n}=0.33$ & $\mathrm{~K}=0.09$ \\
Formulation C & $\mathrm{n}=0.29$ & $\mathrm{~K}=0.09$ \\
\hline
\end{tabular}

Abbreviation: AGnp, andrographolide nanoparticles.
Plant-derived antileishmanials with minimal inherent toxicities are emerging alternatives in chemotherapy of leishmaniasis. $^{24-26}$

The antileishmanial compound AG could provide an excellent low-cost alternative if its problems of solubilization and localization could be solved. AG, an indigenous phytomedicine, can be readily purified from a plant source, A . paniculata. ${ }^{27}$ In addition AG combines immunomodulation and anti-HIV potentials acting via viral tyrosine kinase inhibition. ${ }^{27}$

Solubility characteristics of the test drug and the biopolymer, however, often dictate the choice of nanoparticle preparation technique. Both AG and PLGA were proportionately soluble in chloroform. The role of a stabilizer was observed to be crucial for particle size and success of later cellular uptake for both AG and PLGA. Proportionate application of the stabilizer PVA provided smooth spherical
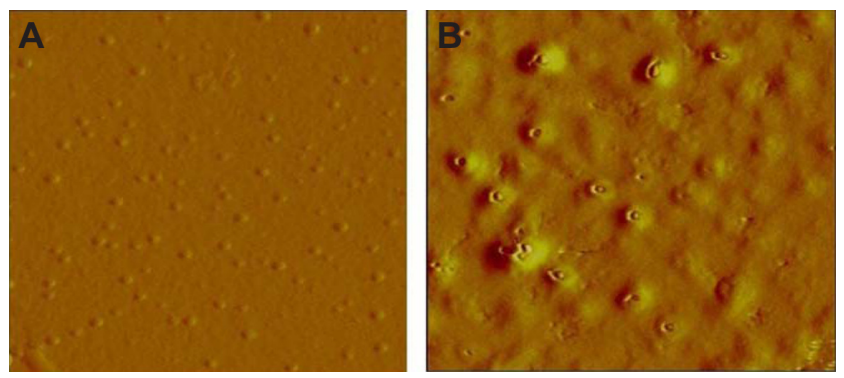

Figure 5 Atomic force microscopy of andrographolide (AG) nanoparticles before and after dissolution. A) Formulation B before release. B) Formulation B, 600 hours after AG release. 
Table 3 Antileishmanial activity of AG, AGnp, and standard antileishmanial agents against $L$. donovani

\begin{tabular}{lccc}
\hline Activity $\left(\mathbf{I C}_{50} \pm \mathbf{S D}\right.$ or $\left.\mathbf{C C}_{50}\right)$ in $\mu \mathrm{M}$ concentrations & & \\
\hline Drug tested & $\begin{array}{l}\text { Axenic amastigote } \\
\left(\mathbf{I C} \mathbf{5 0}_{50} \pm \mathbf{S D}\right)\end{array}$ & $\begin{array}{l}\text { Amastigote in macrophage } \\
\left(\mathbf{I C}_{50} \pm \mathbf{S D}\right)\end{array}$ & $\begin{array}{l}\text { Cytotoxicity assay } \\
\left(\mathbf{C C}_{50}\right)\end{array}$ \\
\hline Formulation A & $64 \pm 8^{*}$ & $54 \pm 5^{*}$ & $>1.2 \times 10^{3}$ \\
Formulation B & $36 \pm 4^{*}$ & $28 \pm 2^{*}$ & $>2 \times 10^{3}$ \\
Formulation C & $76 \pm 6^{*}$ & $62 \pm 8^{*}$ & $>1.8 \times 10^{3}$ \\
Andrographolide & $160 \pm 26$ & $141 \pm 21$ & $>10^{3}$ \\
Stibogluconate & $410 \pm 60^{*}$ & $246 \pm 37^{* *}$ & $>2.4 \times 10^{3}$ \\
Amphotericin B & $0.2 \pm 0.1^{*}$ & $0.15 \pm 0.1^{*}$ & $>13$ \\
\hline
\end{tabular}

Notes: Results expressed as mean \pm SD $(n=3) ;{ }^{*} P<0.01$ significant difference compared with AG; ${ }^{* *} P<0.05$ no significant difference compared with AG. Abbreviations: AG, andrographolide; AGnp, andrographolide nanoparticles; $\mathrm{IC}_{50}$, inhibitory concentration $50 \%$; $\mathrm{CC}_{50}$, cytotoxicity concentration $50 \%$.

nanoparticles having narrower Gaussian size distribution (Table 1). Formulation B nanoparticles showed a negative zeta potential of $-34.8 \mathrm{mV}$, therefore providing prolonged stabilization in suspension, which is required for cellular internalization. Higher drug loading efficiency of up to $80 \% \mathrm{w} / \mathrm{w}$ can be attributed to optimized particulation and entrapment or for polymer and drug chemical interactions. The chemical interactions of AG with the polymer and the stabilizer components were therefore checked in FTIR and no major chemical interaction was observed. AG mass appears ideally localized in particular PLGA nanoparticles. The drug release mechanism for the new AG-PLGA nanoparticles was observed following a diffusion and surface erosion mechanism. This was explained in later AFM studies (Figure 5) showing extensive polymer erosion and drug diffusion. Antileishmanial efficacy of AG was significantly improved in AGnp due to particle characteristics and successful localization of AGnp in infested macrophages, as evidenced by fluorescent nanoparticle internalization (Figure 6). AG loaded in PLGA nanoparticles therefore has reasonable potential

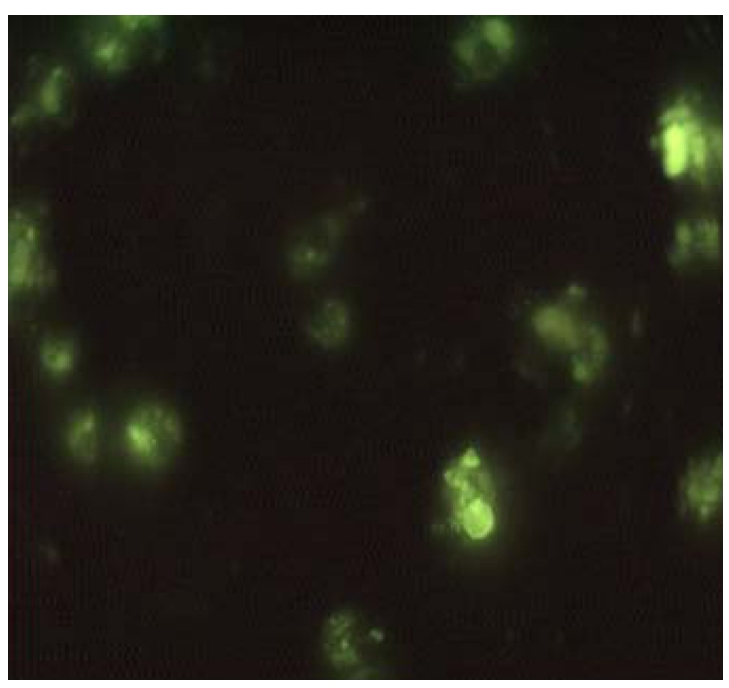

Figure 6 Andrographolide nanoparticles in macrophages under fluorescence microscope after 2 hours' incubation. for further application in chemotherapy of leishmaniasis. Molecularly, the diterpenoid $\mathrm{AG}^{28}$ reacts with active cysteine residues, effectively inducing extrusion of cellular glutathione and converting the intracellular oxy radicals to $\mathrm{H}_{2} \mathrm{O}_{2}$. AG triggers an alternation of intracellular reactive oxygen species and eventually induces collapse of mitochondrial membrane potential. AG was also shown to effectively inhibit mitotic cell division and G2/M arrest in highly proliferating cancerous cells. Earlier work has established some limited successes with liposomal delivery of AG. ${ }^{6}$ Additionally, attempts to deliver an active AG metabolite, 14-deoxy-11 -oxo-andrographolide, using different drug carriers have established an inverse linear relationship between the size of the carriers and antileishmanial efficacy. ${ }^{29}$ A major constraint in antileishmanial chemotherapy is the development of drug resistance implicitly linked to cellular transporters. ${ }^{23}$ Nanoparticle cellular uptake is markedly dependent on size and surface charge. ${ }^{30}$ Nanoparticles in a size range below $200 \mathrm{~nm}$ are associated with increased phagocytosis and can easily localize in macrophages predominantly infested with leishmanial parasite. ${ }^{31}$ The phagolysosomal $\mathrm{pH}$ is acidic around $\mathrm{pH} 4.5$ to 5.0 and the acidic environment has implications in the infested amastigote strategies for faster nutrient acquisition and ion homeostasis. ${ }^{4}$ PLGA, on the other hand, degrades relatively easily at acidic $\mathrm{pH}^{32}$ and the acidic monomers formed further autocatalyze the biodegradation. Size-defined AG nanoparticles in PLGA can therefore boost antileishmanial chemotherapy.

\section{Conclusions}

Limiting parameters for indigenous low-toxicity plantderived chemotherapeutics in leishmaniasis can thus be overcome by nanoparticulation in PLGA followed by drug localization in infected cells of the reticuloendothelial system. Compounds such as AG nanoparticles may provide effective low-cost chemotherapy of leishmaniasis acting through an alternative mechanism. 


\section{Acknowledgments}

Our group would like to thank Mr Anirban Das for his assistance in atomic force microscopy studies. Financial assistance from Department of Biotechnology, Govt. of India and a Senior Research Fellowship to Partha Roy from Indian Council for Medical Research, Govt. of India is gratefully acknowledged.

\section{Disclosure}

The authors declare no conflicts of interest.

\section{References}

1. Cavalli A, Bolognesi ML. Neglected tropical diseases: multitarget-directed ligands in the search for novel lead candidates against Trypanosoma and Leishmania. J Med Chem. 2009;52(23): 7339-7359.

2. Connell DO. Neglected diseases. Nature. 2007;449(7159):157.

3. Ouellette M, Drummelsmith J, Papadopoulou B. Leishmaniasis: drugs in the clinic, resistance and new developments. Drug Resist Updat. 2004;7(4-5):257-266.

4. Croft SL, Coombs GH. Leishmaniasis - current chemotherapy and recent advances in the search for novel drugs. Trends Parasitol. 2003; 19(11):502-508

5. Seifert K, Croft SL. In vitro and in vivo interactions between miltefosine and other antileishmanial drugs. Antimicrob Agents Chemother. 2006;50(1):73-79.

6. Sinha J, Mukhopadhyay S, Das N, Basu MK. Targeting of liposomal andrographolide to L. donovani-infected macrophages in vivo. Drug Deliv. 2000;7(4):209-213.

7. Zhao D, Liao K, Ma X, Yan X. Study of the supramolecular inclusion of $\beta$-cyclodextrin with andrographolide. J Incl Phenom Macrocycl Chem. 2002;43(3-4):259-264.

8. Zhu SP, Kang BA. Distribution and excretion of [35S] $\mathrm{NaHSO}_{3}-$ andrographolide by autoradiography. Acta Pharmacologica Sinica 1981;2(4):266-269.

9. Bala I, Bhardwaj V, Hariharan S, Kharade SV, Roy N, Ravi Kumar MN. Sustained release nanoparticulate formulation containing antioxidantellagic acid as potential prophylaxis system for oral administration J Drug Target. 2006;14(1):27-34.

10. Sarmento B, Ribeiro AJ, Veiga F, Ferreira DC, Neufeld RJ. Insulin-loaded nanoparticles are prepared by alginate ionotropic pregelation followed by chitosan polyelectrolyte complexation. J Nanosci Nanotechnol. 2007;7(8):1-9.

11. Chakraborty P, Das PK. Role of mannose/N-acetylglucosamine receptors in blood clearance and cellular attachment of Leishmania donovani. Mol Biochem Parasitol. 1988;28(1):55-62.

12. Ghosh AK, Bhattacharya FK, Ghosh DK. Leishmania donovani: amastigote inhibition and mode of action of berberine. Exp Parasitol. 1985;60(3):404-413.

13. Debrabant A, Joshi MB, Pimenta PF, Dwyer D. Generation of Leishmania donovani axenic amastigotes: their growth and biological characteristics. Int J Parasitol. 2004;34(2):205-217.

International Journal of Nanomedicine

\section{Publish your work in this journal}

The International Journal of Nanomedicine is an international, peerreviewed journal focusing on the application of nanotechnology in diagnostics, therapeutics, and drug delivery systems throughout the biomedical field. This journal is indexed on PubMed Central, MedLine, CAS, SciSearch ${ }^{\circledR}$, Current Contents ${ }^{\circledR} /$ Clinical Medicine,
14. Senero D, Lemesra JL. Axenically cultured amastigote forms as an in-vitro model for investigation of antileishmanial agents. Antimicrob Agents Chemother. 1997;41(5):972-976.

15. Callahan H, Kelley C, Peretra T, Grogle M. Microtubule inhibitors: structure-activity analysis suggest rational models to identify potentially active compounds. Antimicrob Agents Chemother. 1996;40(4): 947-952.

16. Chang K. Human cutaneous leishmaniasis in a mouse macrophage line: propagation and isolation of intracellular parasites. Science. 1980;209(4462):1240-1244.

17. Freitas MN, Marchetti JM. Nimesulide PLA microspheres as a potential sustained release system for the treatment of inflammatory diseases. Int J Pharm. 2005;295(1-2):201-211.

18. Budhian A, Siegel SJ, Winey KI. Haloperidol-loaded PLGA nanoparticles: Systemic study of particle size and drug content. Int J Pharm. 2007;336(2):367-375.

19. Bellamy LJ. The Infrared Spectra of Complex Molecules. 2nd ed, Pt 1. New York: Chapman and Hall; 1980:30-56.

20. Mukherjee B, Santra K, Patttanaik G, Ghosh S. Preparation, characterization and in-vitro evaluation of sustained release protein-loaded nanoparticles based on biodegradable polymers. Int $J$ Nanomed. 2008;3(4):487-496.

21. Korsemeyer RW, Gurny R, Doelker EM, Buri P, Peppas NA. Mechanism of solute release from porous hydrophilic polymers. Int J Pharm. 1983;15(1):25-35.

22. Grattard N, Pernin M, Marty B, Roudaut G, Champion D, Meste ML. Study of release kinetics of small and high molecular weight substances dispersed into spray-dried ethylcellulose microspheres. J Control Rel. 2002;84(3):125-135.

23. Ponte-Sucre A. Physiological consequences of drug resistance in Leishmania and their relevance for chemotherapy. Kinetoplastid Biol Dis. 2003;2(1):1-10.

24. Lala S, Pramanik S, Mukhopadhay S, Bandhopadhyay S, Basu MK. Harmine: evaluation of its antileishmanial properties in various vesicular delivery systems. J Drug Target. 2004;12(3):165-175.

25. Cruz LJ, Luque-Ortega JR, Rivas L, Albericio F. Kahalalide F. An antitumor depsipeptide in clinical trials, and its analogues as effective antileishmanial agents. Mol Pharm. 2009;6(3):813-824.

26. Habtemariam S. In vitro antileishmanial effects of antibacterial diterpenes from two Ethiopian Premna species: P. schimperi and P. oligotricha. BMC Pharmacol. 2003;3(6):1-6.

27. Mishra SK, Sangwan NS, Sangwan RS. Andrographis paniculata (Kalmegh): A Review. Pharmacognosy Reviews. 2007;1(2):283-298.

28. Cava MP, Chan WR, Haynes LJ, Johnson LF, Weinstein B. The structure of andrographolide. Tetrahedron. 1962;18(4):397-403.

29. Lala S, Nandy AK, Mahato SB, Basu MK. Delivery in vivo of 14-deoxy-11-oxoandrographolide, an antileishmanial agent, by different drug carriers. Indian J Biochem Biophys. 2003;2:169-174.

30. Win KY, Feng SS. Effects of particle size and surface coating on cellular uptake of polymeric nanoparticles for oral delivery of anticancer drugs. Biomaterials. 2005;26(15):2713-2722.

31. Vasir JK, Reddy MK, Labhasetwar VD. Nanosystems in drug targeting: Opportunities and Challenges. Curr Nanosci. 2005;1(1):47-64.

32. Mundargi RC, Babu VR, Rangaswamy V, Patel P, Aminabhavi TM. Nano/micro technologies for delivering macromolecular therapeutics using poly(D,L-lactide-co-glycolide) and its derivatives. $J$ Control Release. 2008;125(3):193-209. 